\title{
Los huevos falsos (SAGs) facilitan el comportamiento social de emergencia en las crías de la tortuga laúd Dermochelys coriacea (Testudines: Dermochelyidae)
}

\author{
Juan Patiño-Martinez ${ }^{1,2}$, Adolfo Marco ${ }^{1}$, Liliana Quiñones ${ }^{1} \&$ Cecilia P. Calabuig ${ }^{1}$ \\ 1. Estación Biológica de Doñana, CSIC, Departamento de conservación de la biodiversidad, Avda. Américo Vespucio \\ s/n, la Cartuja, Sevilla, España; juanpatino@ebd.csic.es, amarco@ebd.csic.es, 1hili@yahoo.es, \\ cecicalabuig@ebd.csic.es \\ 2. Sociedad de ciencias ARANZADI, Departamento de vertebrados, Zorroagagaina, 11 Donostia-San Sebastián.
}

\author{
Recibido 16-VIII-2009. Corregido 08-I-2010. Aceptado 09-II-2010.
}

\begin{abstract}
False eggs (SAGs) facilitate social post-hatching emergence behaviour in Leatherback turtles Dermochelys coriacea (Testudines: Dermochelyidae) nests. Hatchling emergence to the beach surface from deep sand nests occurs without parental care. Social behaviour among siblings is crucial to overcome this first challenge in sea turtles life. This study, carried out at the Caribbean coast of Colombia, describes the emergence social behaviour of hatchlings from eight nests, and assess the nests translocation effects on temporal patterns of emergence. For the first time, we propose that space released by dehydration of shelled albumen globes (SAGs) at the top of the clutch, might be a reproductive advantage, while facilitating neonates to group together in a very limited space, and favouring the synchrony of emergence. The mean time of groups emergence was of 3.3 days, varying between 1 and 6 days. We found that relocation of the nests did not significantly affect the temporal pattern of emergence, which was mainly nocturnal ( $77.7 \%$ of natural nests and $81.7 \%$ of translocated ones). The maximum number of emergences to the surface occurred at the lowest air temperatures (22:00h-06:00h). The selective advantage of this pattern is probably related to the greater rate of predation and mortality by hyperthermia observed during the day. Rev. Biol. Trop. 58 (3): 943-954. Epub 2010 September 01.
\end{abstract}

Key words: tropical nesting beaches, leatherback, hatchlings behaviour, survival, emergence success, SAGs, sea turtles.

Los nidos de la tortuga laúd, Dermochelys coriacea (Vandelli, 1761), contienen generalmente un $70 \%$ de huevos con yema y un $30 \%$ de falsos huevos sin yema (Chacon-Chaverri 1999, Reina et al. 2002, Chacon-Chaverri \& Eckert 2007, Quinones et al. 2007). Estos huevos falsos sin yema son de menor tamaño que los huevos fértiles, están compuestos casi exclusivamente por albúmina y se les conoce como glóbulos de albúmina con cáscara o "SAGs" (shelled albumen globes) (Wallace et al. 2004). Los SAGs tienen la cáscara muy fina, formas irregulares y son depositados generalmente al final de la puesta. Si bien la función adaptativa de estos SAGs no está aun bien establecida, aparentemente tienen efectos positivos sobre el éxito de eclosión de las nidadas en presencia de algunos depredadores (Dutton \& McDonald 1995, Caut et al. 2006). También se les ha reconocido posibles funciones favorables para el intercambio gaseoso y el mantenimiento de la humedad del nido (Dutton \& McDonald 1995).

Las nidadas (huevos fértiles y SAGs) se incuban durante aproximadamente 60 días, en nidos con profundidades entre $0.6 \mathrm{~m}$ y $1.0 \mathrm{~m}$ (Whitmore \& Dutton 1985), en las condiciones ambientales propias de las playas tropicales 
(Chacon-Chaverri et al. 1996). Tras la eclosión, se inicia el proceso de emergencia, en el cual los neonatos ascienden desde el fondo del nido hasta la superficie de la arena. Inicialmente los neonatos permanecen un tiempo variable (días) en la cámara del nido y durante este tiempo ocurre la absorción de buena parte del vitelo y el estiramiento del caparazón y el plastron (Godfrey \& Mrosovsky 1997). El intervalo empleado en el comportamiento de emergencia puede depender de la compactación o la temperatura de la arena (Drake \& Spotila 2002), la profundidad del nido, la sincronía en la eclosión, el número de crías o de la especie (Mrosovsky 1968, Witherington et al. 1990, Moran et al. 1999).

Finalmente, las crías que emergen reptan hasta el océano, guiadas fundamentalmente por señales visuales (Godfrey \& Barreto 1995, Lorne \& Salmon 2007, Kawamura et al. 2009). La inevitable exposición visual u olfativa a depredadores, las temperaturas extremas o la deshidratación son riesgos presentes durante la emergencia y dispersión desde el nido hasta el mar (Mrosovsky 1968, Witherington et al. 1990, Stancyk 1995). En algunos reptiles existe el cuidado de huevos y crías por al menos uno de los padres (Platt et al. 2008), sin embargo, en tortugas marinas ninguna de estas fases cuenta con ayuda parental. Por lo tanto, la rapidez, la sincronía y el éxito en la emergencia tienen importancia biológica ya que influyen la supervivencia inicial de las crías.

Diferentes estudios han abordado el comportamiento de emergencia, abarcando aspectos descriptivos (Carr \& Hirth 1961, Witherington et al. 1990, Glen et al. 2005), tiempos empleados en la emergencia (Christens 1990, Godfrey \& Mrosovsky 1997, Koch et al. 2008), factores medioambientales (Drake \& Spotila 2002, Matsuzawa et al. 2002, Glen et al. 2006), energéticos (Dial 1987, Jones et al. 2007) y de uso de la playa (Kudo et al. 2003). Sin embargo, existen pocos estudios cuantitativos y del efecto de los SAGs que se correlacionen con la emergencia de las crías de la tortuga laúd (Godfrey \& Mrosovsky 1997).
Los programas de traslocación y protección de nidos ex situ, en especies críticamente amenazadas, se consideran en general muy valiosos para garantizar el reclutamiento de crías de la población (Lum 2005, Mazaris et al. 2009), pero podrían estar alterando los patrones de emergencia natural debido a la alteración de la forma, profundidad y compactación de los nidos (Adam et al. 2007). Sin embargo, la información científica en esta materia y en la especie de estudio es actualmente muy limitada.

El propósito de este trabajo fue estudiar el proceso de emergencia exhibido por las crías de $D$. coriacea y compararlo con los reportes previos de otras especies de tortugas marinas, $\mathrm{y}$, evaluar el efecto del traslado de los nidos sobre el patrón temporal de emergencia a la superficie de la playa. Finalmente, de acuerdo con observaciones directas, se propone la función adaptativa de los SAGs como facilitadores del proceso de emergencia en la especie con los nidos más profundos y con menor éxito de eclosión, entre las tortugas marinas (Bell et al. 2004, Tapilatu \& Tiwari 2007).

\section{MATERIALES Y MÉTODOS}

Área de estudio: El estudio se desarrolló durante los años 1998, 2005, 2006 y 2007 al Noroeste de Colombia, en una sección de $3 \mathrm{~km}$ de la playa "Playona" cuya longitud total es de $12 \mathrm{~km}$ ( $8^{\circ} 25^{\prime} 57^{\prime \prime}-8^{\circ} 28^{\prime} 16^{\prime \prime} \mathrm{N}, 77^{\circ} 09^{\prime} 59^{\prime}$ "$77^{\circ} 14^{\prime} 58^{\prime \prime}$ W, Fig. 1) que se encuentra ubicada en Acandí, Chocó. La playa se caracteriza por arena de grano muy fino y altos valores de humedad y compactación con otras playas de la zona (Patiño-Martinez et al. 2008).

Influencia de los SAGs: Con el propósito de calcular el volumen ocupado por los SAGs dentro de la cámara del nido, se contabilizaron todos los huevos y los SAGs de un total de 80 nidos estudiados en la temporada reproductiva de 1998. Se registró el diámetro (Digital caliper Cen-Tech $\mathrm{d}=0.01 \mathrm{~mm} ; \max =150 \mathrm{~mm})$ de una muestra de ocho SAGs por cada nido.

Se usaron los valores medios del diámetro para calcular el volumen (esfera) y se usó el 


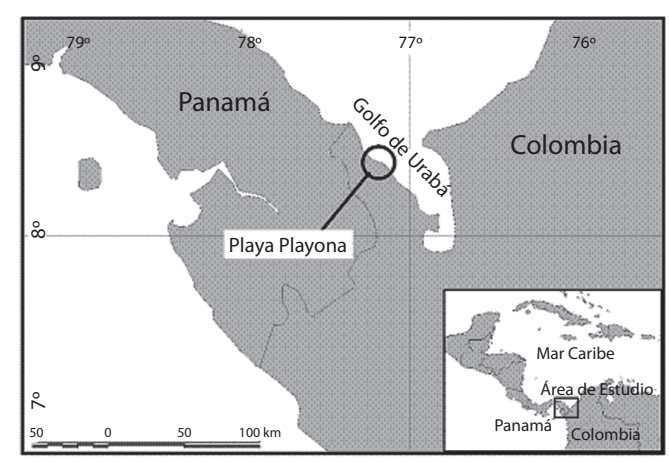

Fig. 1. Ubicación de la playa de estudio.

Fig. 1. Study beach location.

número medio de SAGs por nido para la estima del volumen total ocupado dentro de la nidada. Se estimó la altura potencial de la cámara de aire dejada por los SAGs en función del diámetro de la columna de ascenso, para lo cual se aplicó la fórmula del volumen del cilindro.

Traslado de las puestas: La zona de estudio presenta una alta depredación de las puestas tanto por humanos como por perros domésticos. Con el fin de proteger eficazmente las nidadas seleccionadas, se usó una metodología estándar de traslado (Chacon-Chaverri \& Eckert 2007) desde los lugares naturales de desove elegidos por la hembra, hacia tres corrales de protección $(15 \mathrm{mx} 8 \mathrm{~m})$ en zonas sin inundación intermareal de la playa de estudio. Las nidadas (81 en 1998, 109 en 2005, 2 en 2006 y 5 en 2007) fueron recogidas en bolsas plásticas nuevas, directamente de la cloaca de la hembra y fueron transportadas manualmente y caminando en un lapso menor a 40min hasta los nuevos nidos excavados a $75 \mathrm{~cm}$ de profundidad (Hirth \& Ogren 1987). El desplazamiento máximo fue siempre inferior a $1 \mathrm{~km}$. Una vez trasladados, los nidos no sufrieron ninguna manipulación posterior hasta la eclosión, por tanto, las condiciones ambientales de radiación solar, humedad, tipo de arena o grado de compactación del substrato de incubación fueron los propios de la playa de anidación.
Comportamiento de emergencia: Con el fin de estudiar la evolución del proceso de eclosión y emergencia, se acondicionaron ocho nidos: 1 en 1998, 2 en 2006 y 5 en 2007. A cada uno de ellos se le acopló una lámina de metacrilato transparente $(80 \mathrm{~cm}$ de profundidad, $30 \mathrm{~cm}$ de ancho y $0.2 \mathrm{~cm}$ de grosor) en una de las caras verticales del nido (Fig. 2). La lámina de metacrilato fue previamente enmarcada con dos piezas de madera de $90 \mathrm{~cm}$ de largo, $10 \mathrm{~cm}$ de ancho y $2 \mathrm{~cm}$ de grosor en el eje longitudinal y dos más de $45 \mathrm{~cm}$ de largo, $5 \mathrm{~cm}$ de ancho y $2 \mathrm{~cm}$ de grosor en el eje transversal. Este dispositivo se enterró al menos veinte días antes de iniciar la traslocación de nidos, removiendo la arena del exterior del futuro nido.

A partir del inicio de la última semana de los 60 días estimados de duración media de la incubación (Duque et al. 2000), se visualizó diariamente el interior de los nidos retirando la arena exterior de la lámina transparente. Se describieron todas las observaciones del comportamiento desde la eclosión hasta la emergencia. Para evitar la entrada de insectos al nido

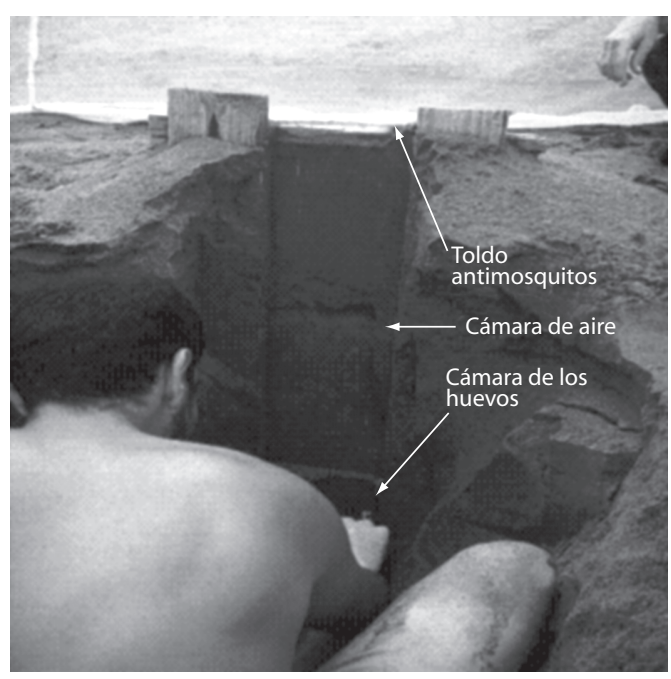

Fig. 2. Nido acondicionado para la observación de la emergencia. Se indican las cámaras de aire y de los huevos, $\mathrm{y}$ el toldo anti-mosquitos.

Fig. 2. Experimental nest for the emergence study. Nest, air chambers and anti-mosquitos owning are indicated. 
durante esta fase del estudio, se acondicionó un habitáculo con un toldo protector antimosquitos de $4 \mathrm{~m} \times 3 \mathrm{~m} \times 2 \mathrm{~m}$ (largo $\mathrm{x}$ ancho $\mathrm{x}$ alto) sobre cada nido (Fig. 2). Se calculó la duración de la emergencia, como el periodo transcurrido desde los primeros movimientos en grupo en la cámara del nido hasta la emergencia definitiva a la superficie del primer grupo. Se calculó también la velocidad máxima de ascenso, como la máxima distancia recorrida en los periodos de actividad observados.

Registro de temperaturas: Con el fin de conocer las temperaturas experimentadas por las crías durante el proceso de emergencia, se registraron las temperaturas del aire y de la arena de la playa a $40 \mathrm{~cm}, 50 \mathrm{~cm}$ y $75 \mathrm{~cm}$ de profundidad. Para lo cual se usaron registradores automáticos de temperatura cilíndricos de $3.0 \mathrm{~cm}$ de base $\mathrm{x} 1.7 \mathrm{~cm}$ de altura (dataloggers Hobo StowAway TidbiT v2 Onset $\pm 0.2^{\circ} \mathrm{C}$ precisión), programados para recoger información a intervalos de $0.5 \mathrm{~h}$, durante el periodo de eclosiones comprendido desde Mayo-6 hasta Mayo-26 de 2006.

Patrón temporal de emergencia: Con el fin de evaluar el efecto del traslado de los nidos sobre la distribución temporal de las emergencias a la superficie de la arena, se registraron datos de un total de 109 nidos trasladados y 18 nidos naturales in situ (sin trasladar), que fueron identificados por triangulación con postes ubicados en la zona posterior de la playa. Durante el periodo 17 de Mayo-28 de Junio de 2005 , la playa fue monitoreada de forma continua por seis grupos de asistentes de campo, en dos turnos desde las 17:00h hasta las 02:00h y desde las 05:00h hasta las 10:00h. Diferentes inspecciones de los nidos fueron realizadas diariamente al azar durante las horas diurnas, para completar el estudio.

Se registró la hora exacta de emergencia, por la observación directa de las crías dentro de una malla de protección circular $(75 \mathrm{~cm}$ de diámetro x $50 \mathrm{~cm}$ de altura) enterrada a $25 \mathrm{~cm}$ de profundidad. La malla fue puesta sobre cada nido el día 45 incubación. Se incluyó cualquier emergencia detectada al inicio de la prospección de las 05:00h, como ocurrida en el periodo 02:00h-05:00h que transcurrió sin observadores. Se consideraron emergencias nocturnas todas las ocurridas entre las 18:00h y las 06:00h. Con el objeto de analizar la distribución temporal durante el periodo nocturno se consideró el horario de anochecer entre las $18: 00 \mathrm{~h}$ y las $00: 00 \mathrm{~h}$ y el de madrugada entre las 00:00h y las 06:00h.

\section{RESULTADOS}

Influencia de los SAGs: Los nidos estudiados de tortuga laúd tuvieron una media de 81.0 huevos con yema (D.E.=19.1) y 34.3 (D.E.=15.5) SAGs por nido. Todos los nidos presentaron SAGs. El diámetro medio de los huevos fue de $53.6 \mathrm{~mm}$ (I.C. $95 \%=53.2-54.1$ ) y de $35.0 \mathrm{~mm}$ (I.C.95\%=34.1-35.9) para los SAGs. El volumen medio ocupado por los SAGs en la nidada fue de $785 \mathrm{~cm}^{3}$. La altura estimada de la cámara de aire proporcionada por los SAGs dentro de la columna de arena fluctuó entre $2.5 \mathrm{~cm}$ y $4.5 \mathrm{~cm}$ para diámetros del cilindro de ascenso entre $20 \mathrm{~cm}$ y $15 \mathrm{~cm}$ respectivamente. Este volumen ocupado por los SAGs se reemplazó parcialmente por aire cuando los SAG se deshidrataron dentro de la cámara del nido, facilitando la movilidad y el agrupamiento de las crías tras la eclosión.

Comportamiento de emergencia: Las primeras crías eclosionadas se ubicaron encima de la nidada y debajo de la columna de arena y esperaron para agruparse con las crías de sucesivas eclosiones. Desde el inicio de la eclosión se observó un espacio de aire sobre la cámara de huevos disponible para las primeras crías eclosionadas. Este espacio inicial se formó principalmente por la previa deshidratación de los SAGs y aumentó gradualmente con las sucesivas eclosiones que liberaron fluidos y aplastaron las cáscaras. El nido 5 fue descartado de los análisis por su nulo éxito de eclosión (Cuadro 1).

El movimiento inicial de las crías agrupadas provocó el desprendimiento de una capa 
CUADRO1

Observaciones de la emergencia en ocho nidos de tortuga laúd, D. coriacea

TABLE 1

Emergence behaviour observations in eight leatherback turtle nests, D. coriacea

$\begin{array}{ccccccccc}\text { Nido } & \begin{array}{c}\text { Fecha de } \\ \text { puesta }\end{array} & \begin{array}{c}\text { Fecha de } \\ \text { eclosión }\end{array} & \begin{array}{c}\text { Fecha de } \\ \text { emergencia }\end{array} & \begin{array}{c}\text { Número } \\ \text { de } \\ \text { huevos }\end{array} & \begin{array}{c}\text { Número de } \\ \text { SAGs }\end{array} & \begin{array}{c}\text { Número } \\ \text { de crías } \\ \text { emergentes }\end{array} & \begin{array}{c}\text { Éxito de } \\ \text { eclosión } \\ (\%)\end{array} & \begin{array}{c}\text { Duración } \\ \text { emergencia } \\ \text { (horas) }\end{array} \\ 1 & 22 / 04 / 98 & \mathrm{n} / \mathrm{o} & 20 / 06 / 98 & 65 & 58 & 55 & 84.6 & 25 \\ 2 & 20 / 03 / 06 & 18 / 05 / 06 & 22 / 05 / 06 & 85 & 15 & \mathrm{n} / \mathrm{o} & \mathrm{n} / \mathrm{o} & 96 \\ 3 & 26 / 03 / 06 & 25 / 05 / 06 & 27 / 05 / 06 & 69 & 44 & 30 & 43.5 & 72 \\ 4 & 16 / 03 / 07 & 12 / 05 / 07 & 15 / 05 / 07 & 94 & 7 & 14 & 14.9 & 72 \\ 5 & 16 / 03 / 07 & \mathrm{n} / \mathrm{o} & \mathrm{n} / \mathrm{o} & 82 & 25 & 0 & 0 & \mathrm{n} / \mathrm{o} \\ 6 & 22 / 03 / 07 & 20 / 05 / 07 & 22 / 05 / 07 & 101 & 25 & 28 & 48.3 & 70 \\ 7 & 22 / 03 / 07 & 21 / 05 / 07 & 26 / 05 / 07 & 60 & 15 & 24 & 40.0 & 144 \\ 8 & 26 / 03 / 07 & \mathrm{n} / \mathrm{o} & 29 / 05 / 07 & 79 & 37 & 22 & 27.8 & \mathrm{n} / \mathrm{o}\end{array}$

$\mathrm{n} / \mathrm{o}=$ no observada.

de arena del techo de la cámara sobre las crías. La columna de arena sobre los huevos se fragmentó formando una o dos cámaras de aire en medio de la columna de arena (entre 10 y $35 \mathrm{~cm}$ de profundidad desde la superficie, Fig. 2). La amplitud de cada cámara varió durante la emergencia desde $3 \mathrm{~cm}$ hasta $15 \mathrm{~cm}$ (Fig. 2). Además, se hizo perceptible un ligero hundimiento de la arena superficial de la playa, apreciable desde el exterior justo encima de los nidos. En todos los casos este hundimiento fue producto del movimiento de ascenso de las crías y dependió del momento en que se desprendió la capa superior de la columna de arena. En el nido sin eclosión no se observó el desprendimiento de la columna de arena, ni el hundimiento de la arena superficial.

El comportamiento de ascenso tuvo un patrón estereotipado de cooperación en grupo, dependiente de la ubicación de cada individuo. Así, las tortugas de la capa superior del grupo iniciaron el movimiento convulsivo de aletas y cabeza provocando el desprendimiento de una capa de arena en la parte superior de la cámara del nido. Las de los lados rozaron las paredes de arena con movimientos frenéticos de las aletas en sentido vertical y las del fondo pisotearon y compactaron la arena que se filtró desde arriba por entre los cuerpos. Este comportamiento ocurrió en episodios de ascenso regulares o "frenesí de grupo" con periodos de descanso intercalados. El grupo de crías se acercó gradualmente a la superficie por el desplome sucesivo de porciones de arena y la acumulación de esta en el fondo. Se comprobó una extraordinaria regularidad en el inicio de cada frenesí de ascenso en grupo (nido 1, Cuadro 1). El tiempo medio que transcurrió entre el inicio de cada episodio de frenesí fue de $3.6 \mathrm{~min}$ (D.E. $=0.8$, rango $=2.5-5.0 \mathrm{~min}, \mathrm{n}=9$ ). La velocidad máxima de ascenso fluctuó entre nidos y mostró un amplio rango, así: $20 \mathrm{~cm} / \mathrm{h}$ en el nido 1 y $10 \mathrm{~cm} /$ día en el nido 2 (Cuadro 1). El intervalo de tiempo promedio empleado en la emergencia fue de 3.3 días (D.E.=1.6, rango $=1-6$ días $)$.

La emergencia ocurrió en uno o dos grupos de crías y se observó la imposibilidad de emergencia en solitario de individuos en el fondo de los nidos. Se observó también la rápida aparición de larvas probablemente de dípteros (McGowan et al. 2001, Hall \& Parmenter 2008) en medio de los huevos tras la emergencia y alrededor de las crías que no emergieron en grupo. El comportamiento de emergencia en grupo presentó periodos prolongados de 
inactividad, que coincidieron con el momento de alcanzar la(s) cámara(s) de aire de la columna de arena y justo debajo de la superficie de la playa, donde típicamente esperaron hasta el momento de la emergencia (hasta $23 \mathrm{~h}$ en nido 1, Cuadro 1).

El dispositivo de observación no tuvo efecto significativo sobre el éxito medio de eclosión (t-student=1.99, gl 63, p=0.49), en los nidos eclosionados con dispositivo estuvo entre $14.9 \%$ y $84.6 \%$ (media $=43.2 \%$, D.E. $=23.6)$ y en los nidos sin dispositivo entre 1.2\%-91.7\% (media $=35.3 \%$, D.E. $=26.6$ ). Sin embargo, el tiempo total desde la puesta de los huevos hasta la emergencia definitiva fue menor en los nidos trasladados (media=59.5días, D.E=1.7) con respecto a nidos naturales (media $=61.2$ días, D.E. $=1.5$, t-student $=2.01, \mathrm{gl} 44, \mathrm{p}<0.05)$.

Patrón temporal de emergencia: $\mathrm{La}$ periodicidad diaria de la emergencia de los neonatos fue muy consistente comparativamente entre nidos naturales y trasladados. El 81.6\% de los nidos trasladados y el $77.7 \%$ de los nidos naturales emergieron en el periodo nocturno $\left(\mathrm{Chi}^{2} \mathrm{gl} \mathrm{1}=0.15, \mathrm{p}=0.697\right)$, con el pico de actividad entre las 21:00h-22:00h en ambas condiciones (Fig. 3). Tampoco se observó un patrón diferente de emergencias a lo largo del periodo nocturno, así el 14.28\% de los nidos naturales y el $20.22 \%$ de los nidos trasladados emergieron durante la madrugada (00:00h-06:00h, $\mathrm{Chi}^{2} \mathrm{gl}$ $1=0.27, p=0.602$ ). En general, la actividad de emergencia definitiva a la superficie y el posterior desplazamiento al mar, ocurrió durante los periodos de menor temperatura ambiental del aire $\left(25.3^{\circ} \mathrm{C}\right.$ y $27.0^{\circ} \mathrm{C}$. Figs. 3 y 4$) . \mathrm{El}$ resto de emergencias diurnas, se distribuyó entre 06:00h y $17: 00 \mathrm{~h}$ en nidos trasladados, incluyendo un $3 \%$ entre $12: 00 \mathrm{~h}$ y $16: 00 \mathrm{~h}$ de temperaturas máximas $\left(29.8^{\circ} \mathrm{C}\right.$ y $\left.30.5^{\circ} \mathrm{C}\right)$ y entre $06: 00 \mathrm{~h}$ y $10: 00 \mathrm{~h}$ de temperaturas entre $25^{\circ} \mathrm{C}$ y $28^{\circ} \mathrm{C}$ en nidos naturales (Figs. 3 y 4).

Las temperaturas mínimas del aire en el exterior de los nidos fueron registradas entre las 22:00h y las $06: 00 \mathrm{~h}$, con diferencias significativas en la temperatura media del aire entre las diferentes horas del día (ANOVA $F_{23}$,
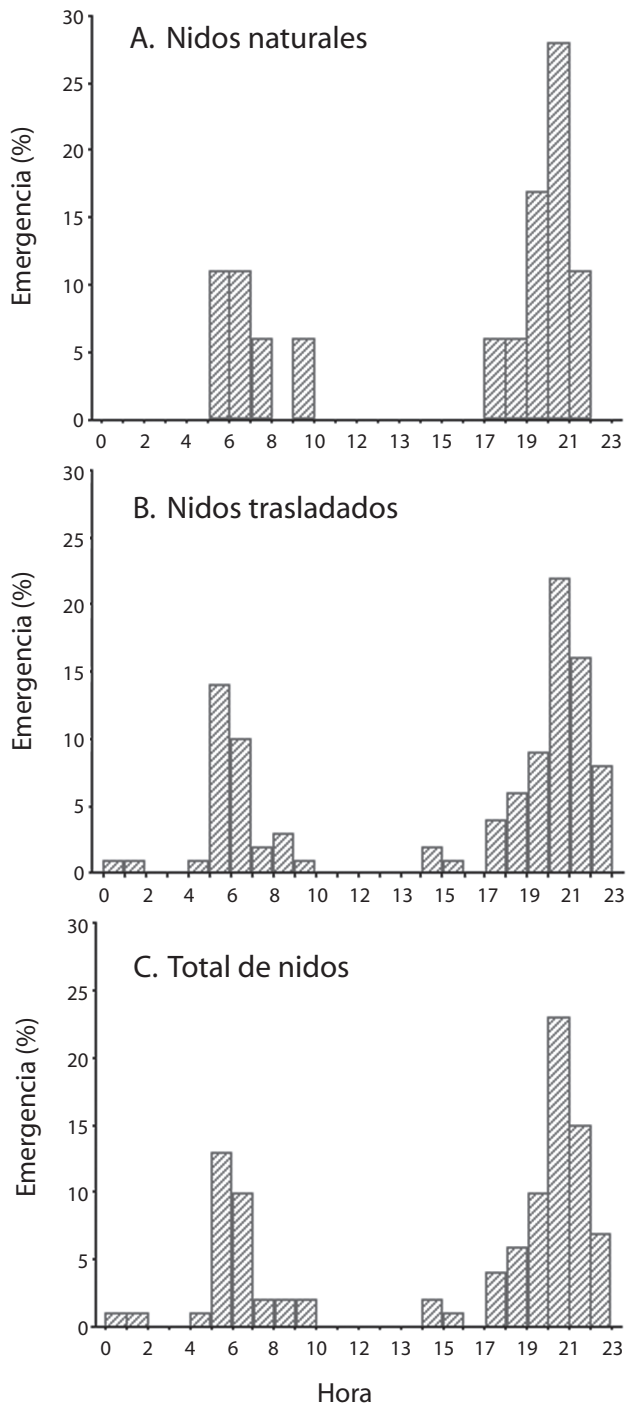

Fig. 3. Distribución temporal de las emergencias a la superficie de la playa en crías de $D$. coriacea, procedentes de nidos naturales (A), trasladados (B) total de los nidos estudiados (C).

Fig. 3 Temporal distribution of emergence at the beach surface for D. coriacea hatchlings, from natural (A), hatchery (B) and total studied nests (C).

${ }_{1224}=162.63, \mathrm{p}<0.0001, \mathrm{n}=2860$, Fig. 4). Por el contrario, la tempe ratura de la arena a profundidades superiores a $40 \mathrm{~cm}$, fluctuó en el estrecho rango entre $29.0^{\circ} \mathrm{C}$ y $30.2^{\circ} \mathrm{C}$ en función de la profundidad (Fig. 4). 


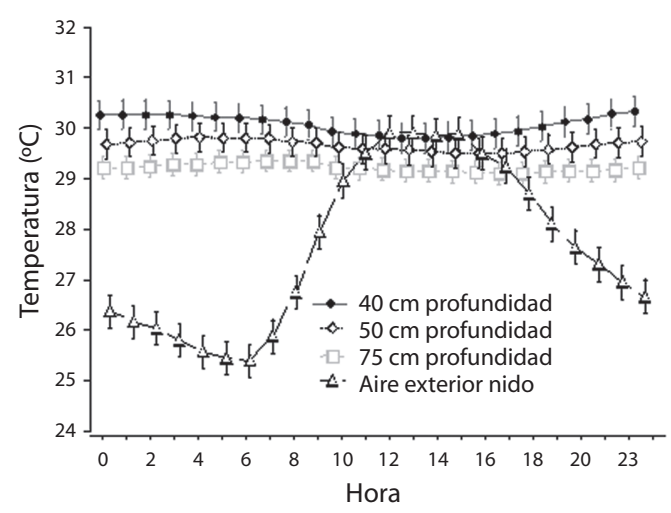

Fig. 4. Variación media diaria de la temperatura del aire en la superficie de los nidos y a tres diferentes profundidades en la arena de la playa.

Fig. 4. Mean daily variation of air temperature at nest surface and at three different sand depths.

\section{DISCUSIÓN}

Influencia de los SAGs: Los SAGs, compuestos básicamente por albumen (agua y proteínas) y cáscara (Wallace et al. 2006), no están fecundados, pero ocupan un volumen importante $\left(700-800 \mathrm{~cm}^{3}\right)$ de las puestas de D. coriacea. La masa media de los SAGs en cada nidada es $1 \mathrm{~kg}$ y representa entre el $13 \%$ y $20 \%$ del peso total de la puesta (Hirth \& Ogren 1987, Wallace et al. 2006). La producción de SAGs, es entonces, un fenotipo paradójico por el costo que representan y la aparente ausencia de beneficio inmediato. Las posibles funciones de los SAGs han sido investigadas (Frazier \& Salas 1984, Dutton \& McDonald 1995, Wallace et al. 2004, Caut et al. 2006), pero permanecen aun sin esclarecer. Se ha informado la posible influencia de los SAGs en el éxito de los nidos, por ejemplo evitando la depredación de los huevos fértiles por grillos del género Scapteriscus spp. (Maros et al. 2005, Caut et al. 2006). Se sugiere que los insectos tienen una mayor probabilidad de encontrar los SAGs y una mayor preferencia por estos cuando se encuentran hidratados. Además se propone que los SAGs deshidratados liberan sustancias que podrían inhibir la depredación. Sin embargo, en zonas donde no existe depredación por grillos, el éxito de eclosión no presenta diferencias significativas entre nidos con o sin SAGs (Dutton \& McDonald 1995). Se ha sugerido también que el efecto barrera en la parte superior de la nidada, favorece el intercambio gaseoso, la amortiguación térmica o el mantenimiento de la humedad al evitar la entrada de arena entre los huevos (Dutton \& McDonald 1995). Sin embargo estudios más recientes rechazan dichas hipótesis, al no encontrar efecto del número total de SAGs sobre los valores mínimos de presión de $\mathrm{O}_{2}$ ni sobre la temperatura en la cámara de los huevos (Wallace et al. 2004).

Nuestros resultados destacan la importancia del agrupamiento inicial de las crías tras la eclosión, para facilitar el comportamiento social de emergencia. Las primeras crías eclosionadas encontrarían una fuerte presión de las paredes de arena y de los huevos sin los espacios de aire facilitados por los SAGs. Inicialmente el efecto barrera descrito en otras de las hipótesis evita que los espacios de aire entre los huevos se colmaten de arena. Posteriormente la deshidratación de los SAGs (Caut et al. 2006) aporta el espacio suficiente en la parte superior de la cámara de los huevos para permitir el movimiento y la agrupación de las crías. Por lo tanto, se sugiere la función adaptativa de los SAGs como facilitadores del proceso de emergencia en grupo, representando una ventaja reproductiva al favorecer la sincronía y posiblemente la supervivencia posterior de las crías. Este tipo de nidada con un volumen importante de SAGs es exclusivamente de tortuga laúd y podría facilitar la emergencia en grupo en los nidos más profundos y con el menor éxito de eclosión entre todas las especies de tortugas marinas (Bell et al. 2004).

Comportamiento de emergencia: Las primeras tortugas eclosionadas esperan y se agrupan en la parte superior de la nidada antes de empezar el trabajo de emergencia coordinado de forma similar al comportamiento previamente descrito para la tortuga verde (Carr \& Hirth 1961). Es posible que exista plasticidad 
en el momento de la eclosión y que huevos incubados en el fondo del nido a temperaturas ligeramente inferiores, aceleren su eclosión (Spencer et al. 2001). Sin embargo, el orden y la posición de los huevos eclosionados y los mecanismos que pueden inducir la sincronía en la eclosión están aún sin determinar y son necesarios más estudios experimentales en esta materia.

En general, el rango y el tiempo medio empleado en la emergencia de tortuga laúd, corresponde con reportes previos para otras especies (Christens 1990, Witherington et al. 1990, Godfrey \& Mrosovsky 1997). Por ejemplo, para Caretta caretta, Chelonia mydas y Eretmochelys imbricata el rango conocido está entre cuatro y siete días (Godfrey \& Mrosovsky 1997). Por el contrario, la velocidad máxima de ascenso de $20 \mathrm{~cm} / \mathrm{h}$ encontrada en uno de los nidos estudiados, no ha sido nunca antes descrita para ninguna especie de tortuga marina.

Hasta la fecha no se ha descrito metodología alguna para distinguir morfológicamente el sexo de las crías y solo es posible mediante la observación directa de las gónadas en crías muertas. Sin embargo, es habitual evitar el sacrificio de ejemplares en peligro crítico de extinción, y en su lugar se recomienda usar la duración del periodo de incubación para la estimación indirecta de la razón de sexos de las crías en la naturaleza (Rimblot et al. 1985, Godley et al. 2002). Así, el conocimiento de la duración de la emergencia es un dato útil para el cálculo de la duración de la incubación en playas (desde la puesta hasta la eclosión) y la comparación con curvas estándar de duración de la incubación y razón de sexos obtenidas en laboratorio (Mrosovsky et al. 2002).

En el presente estudio, la emergencia de las crías de tortuga laúd a la superficie ocurre de forma sincrónica en uno o dos grupos y es probablemente la más sincrónica entre las especies de tortugas marinas (Glen et al. 2005). Estudios previos han demostrado que en general existe, una mayor sincronía en nidos profundos (Houghton \& Hays 2001, Koch et al. 2008). La emergencia sincrónica de un gran número de crías desborda la capacidad de consumo de los depredadores durante la dispersión (perros, aves, cangrejos), induciendo el efecto de dilución del riesgo y en consecuencia mejora la probabilidad individual de alcanzar el mar (Tucker et al. 2008). Sin embargo, aunque en el área de estudio no se ha cuantificado el impacto por depredadores en el mar (peces y aves), aparentemente grupos grandes de crías en el mar podrían verse más afectados por depredación que ejemplares solitarios (Glen et al. 2005, Harewood \& Horrocks 2008).

Varias hipótesis pueden apoyar la necesidad de emergencia grupal y explicar la imposibilidad de ascenso en solitario de crías con eclosión retrasada. Primero, la rápida expansión de larvas de dípteros (McGowan et al. 2001, Hall \& Parmenter 2008) desde los huevos parasitados hacia el resto de la nidada, aumenta sustancialmente el riesgo de muerte por infección a través del remanente del vitelo de las crías rezagadas. Segundo, el ascenso en solitario representa un mayor coste energético, especialmente sin las cámaras de aire, que son usadas por el grupo para descansar y disminuir los niveles de lactato acumulados en sangre y músculo (Dial 1987), y tercero, tras las primeras eclosiones, aumenta sustancialmente la probabilidad de depredación tanto por animales silvestres como domésticos (Glen et al. 2005).

Patrón temporal de emergencia: La periodicidad diaria en los eventos de emergencia sugiere una fuerte selección en contra de la emergencia diurna. Las ventajas de este patrón tienen que ver probablemente con la fuerte relación entre las altas temperaturas de la superficie de la arena durante el día y la disminución de la actividad física de las crías (Janzen et al. 2000, Drake \& Spotila 2002) o incluso el riesgo de muerte por hipertermia (Mrosovsky 1968). Además, en la emergencia diurna ocurre una mayor tasa de depredación (Glen et al. 2006), causada tanto por una mayor actividad de los depredadores como por una mayor conspicuidad de las crías.

Los datos demuestran que la traslocación de nidos a corrales no influye en los patrones temporales de emergencia. La mayoría de las crías emergieron entre las 21:00h y 23:00h en 
ambas condiciones. A pesar de la ausencia de significación estadística, cabe resaltar que sólo en nidos trasladados se registraron emergencias durante las horas de mayor temperatura ambiental (12:00h-16:00h). Eventualmente un porcentaje muy pequeño de nidos naturales también presenta este tipo de emergencias, a pesar de no haber sido detectadas en los nidos del estudio (Patino-Martínez obs. pers.). Por otro lado la traslocación de nidos causó un acortamiento del tiempo total de incubación y emergencia, que pudo deberse a la menor variabilidad en la profundidad de los nidos o una menor compactación de la arena que facilitaría la emergencia. Se podría especular sobre el posible aumento de temperatura en el corral por la acumulación de nidos y el calor metabólico desprendido por ellos, que acortaría el periodo de incubación.

Los registros de temperatura de la arena sugieren que las crías no están expuestas a grandes fluctuaciones, al menos a profundidades iguales o superiores a $40 \mathrm{~cm}$. Sin embargo, la espera variable de tiempo observada bajo la superficie de la playa, puede indicar una respuesta final de emergencia a la superficie inducida por la temperatura ambiental (Bustard 1967). Dos situaciones han sido sugeridas como generadoras del estímulo fisiológico que provoca la emergencia a la superficie: 1) bajar de un límite crítico de temperatura, o 2) rápido decrecimiento de temperaturas en la columna de arena (Moran et al. 1999). Nuestros datos sugieren que las crías de tortuga laúd emergen definitivamente a la superficie de la playa cuando las temperaturas del aire son iguales o inferiores a $29^{\circ} \mathrm{C}$.

En conclusión, este estudio contribuye al conocimiento general del comportamiento de la especie en una fase poco visible de su ciclo vital. El conocimiento de la duración de la emergencia en tortuga laúd contribuye con el ajuste de las estimas indirectas de la razón de sexos en las playas. Estimar la razón de sexos en las crías provenientes de los programas de conservación es esencial para el éxito de dichos programas (Chan \& Liew 1996) y en definitiva para la conservación de especies críticamente amenazadas (Spotila et al. 2000).

Se demuestra también que el traslado de nidos al corral de protección no altera el patrón temporal de emergencia y este parece estar condicionado por la temperatura. Sin embargo, impactos humanos que alteren las características de compactación, profundidad o temperatura en los nidos (tráfico de vehículos, ganado o personas, la remoción o adición de arena a las playas o las urbanizaciones) pueden potencialmente afectar el proceso de incubación y emergencia de las crías.

Se propone por primera vez que el espacio liberado en la parte superior de la nidada por la deshidratación de los falsos huevos o globos de albúmina (SAGs) es aprovechado por las crías eclosionadas para el movimiento inicial y el agrupamiento en un espacio muy limitado. Así, la producción de SAGs constituye una ventaja reproductiva al favorecer la emergencia sincrónica en la especie en estudio que tiene los nidos más profundos y menos exitosos entre las tortugas marinas.

\section{AGRADECIMIENTOS}

Agradecemos al grupo de investigadores locales de Acandí y Playona (GILA) por su apoyo en la traslocación de los nidos, el mantenimiento del corral y el registro de datos de campo. Asimismo a la Autoridad Ambiental Codechocó y a los Consejos Mayores de comunidades negras de Acandí, por facilitar los permisos de investigación. La financiación para el estudio fue otorgada por la Junta de Andalucía y la Fundación BBVA de España. Agradecemos también la revisión y los comentarios de dos evaluadores anónimos que ayudaron a mejorar el manuscrito.

\section{RESUMEN}

La emergencia de las crías de tortuga laúd eclosionadas en los nidos profundos desde la arena hasta la superficie de la playa ocurre sin ayuda parental y es el primer gran desafío de supervivencia en su ciclo de vida. Este estudio, desarrollado en la costa Caribe colombiana, describe el 
comportamiento social de emergencia de neonatos y evalúa el efecto de la traslocación de los nidos en los patrones temporales de emergencia. Se propone por primera vez que el espacio liberado por la deshidratación de falsos huevos (SAGs) en la nidada, representa una ventaja reproductiva al facilitar el agrupamiento de los neonatos en un espacio muy limitado y favorecer la sincronía de la emergencia. El tiempo medio registrado para la emergencia en grupo fue de 3.3 días, variando entre uno y seis días. La traslocación de los nidos no afectó el patrón temporal de emergencia que fue predominantemente nocturno $(77.77 \%$ en nidos naturales y $81.65 \%$ en trasladados). Los picos máximos de emergencias a la superficie coincidieron con los periodos de menor temperatura ambiental exterior (22:00h-06:00h). La ventaja selectiva de este patrón temporal y de la emergencia sincrónica está probablemente relacionada con las mayores tasas de depredación y mortalidad por hipertermia observadas durante el día.

Palabras clave: Playas tropicales, Caná, comportamiento, éxito de emergencia, huevos, tortugas marinas, Colombia.

\section{REFERENCIAS}

Adam, V., C. Tur, A.F. Rees \& J. Tomas. 2007. Emergence pattern of loggerhead turtle (Caretta caretta) hatchlings from Kyparissia Bay, Greece. Mar. Biol. 151: 1743-1749.

Bell, B.A., J.R. Spotila, F.V. Paladino \& R.D. Reina. 2004. Low reproductive success of leatherback turtles, Dermochelys coriacea, is due to high embryonic mortality. Biol. Conserv. 115: 131-138.

Bustard, H.R. 1967. Mechanism of nocturnal emergence from nest in Green turtle hatchlings. Nature 214: 317.

Carr, A. \& H. Hirth. 1961. Social facilitation in green turtle siblings. Anim. Behav. 9: 68-70.

Caut, S., E. Guirlet, P. Jouquet \& M. Girondot. 2006. Influence of nest location and yolkless eggs on the hatching success of Leatherback turtle clutches in French Guiana. Can. J. Zool. 84: 908-915.

Chacon-Chaverri, D. 1999. Dermochelys coriacea (Testudines : Dermochelyidae) nesting in playa Gandoca, Costa Rica (1990 through 1997). Rev. Biol. Trop. 47: 225-236.

Chacon-Chaverri, D. \& K.L. Eckert. 2007. Leatherback sea turtle nesting at Gandoca Beach in Caribbean Costa Rica: Management recommendations from fifteen years of conservation. Chelonian Conserv. Bi. 6: 101-110.
Chacon-Chaverri, D., W. McLarney, C. Ampie \& B. Venegas. 1996. Reproduction and conservation of the leatherback turtle Dermochelys coriacea (Testudines: Dermochelyidae) in Gandoca, Costa Rica. Rev. Biol. Trop. 44: 853-860.

Chan, E.H. \& H.C. Liew. 1996. Decline of the Leatherback population in Terengganu, Malaysia, 1956-1995. Chelonian Conserv. Bi. 2: 196-203.

Christens, E. 1990. Nest emergence lag in Loggerhead sea turtles. J. Herpetol. 24: 400-402.

Dial, B.E. 1987. Energetics and performance during nest emergence and the hatchling frenzy in Loggerhead sea-turtles (Caretta-Caretta). Herpetologica 43: $307-$ 315.

Drake, D.L. \& J.R. Spotila. 2002. Thermal tolerances and the timing of sea turtle hatchling emergence. J. Therm. Biol. 27: 71-81.

Duque, V.M., V.P. Páez \& J. Patiño-Martínez. 2000. Ecología de anidación y conservación de la tortuga caná, (Dermochelys coriacea), en La Playona, Golfo de Urabá Chocoano (Colombia), en 1998. Act. Biol. 22: 37-53.

Dutton, P. \& D. McDonald. 1995. Hatch rates of leatherback (Dermochelys coriacea) clutches reburied with and without yolkless eggs. Proceedings of the 12th Annual Symposium on Sea Turtle Biology and Conservation.U.S. Dept. Commerce. NOAA Tech. Memo. NMFS-SEFSC-361, pp. 177-178. Jekyll Island, Georgia, EEUU.

Frazier, J. \& S. Salas. 1984. The status of marine turtles in the Egyptian Red-Sea. Biol. Conserv. 30: 41-67.

Glen, F., A.C. Broderick, B.J. Godley \& G.C. Hays. 2005. Patterns in the emergence of green (Chelonia mydas) and loggerhead (Caretta caretta) turtle hatchlings from their nests. Mar. Biol. 146: 1039-1049.

Glen, F., A.C. Broderick, B.J. Godley \& G.C. Hays. 2006. Thermal control of hatchling emergence patterns in marine turtles. J. Exp. Mar. Biol. Ecol. 334: 31-42.

Godfrey, M.H. \& R. Barreto. 1995. Beach Vegetation and seafinding orientation of turtle hatchlings. Biol. Conserv. 74: 29-32.

Godfrey, M.H. \& N. Mrosovsky. 1997. Estimating the time between hatching of sea turtles and their emergence from the nest. Chelonian Conserv. Bi. 2: 581-585.

Godley, B.J., A.C. Broderick, F. Glen \& G.C. Hays. 2002. Temperature-dependent sex determination of Ascen- 
sion Island green turtles. Mar. Ecol. Prog. Ser. 226: 115-124.

Hall, S.C.B. \& C.J. Parmenter. 2008. Necrotic egg and hatchling remains are key factors attracting dipterans to sea turtle (Caretta caretta, Chelonia mydas, Natator depressus) nests in Central Queensland, Australia. Copeia 75-81.

Harewood, A. \& J. Horrocks. 2008. Impacts of coastal development on hawksbill hatchling survival and swimming success during the initial offshore migration. Biol. Conserv. 141: 394-401.

Hirth, H. \& L. Ogren. 1987. Some aspects of the ecology of the Leatherback turtle Dermochelys coriacea at Laguna Jalova, Costa Rica. U.S Department of commerce. National Oceanic and Atmospheric Administration NOAA. Malcolm Baldrige, EEUU.

Houghton, J.D.R. \& G.C. Hays. 2001. Asynchronous emergence by loggerhead turtle (Caretta caretta) hatchlings. Naturwissenschaften 88: 133-136.

Janzen, F.J., J.K. Tucker \& G.L. Paukstis. 2000. Experimental analysis of an early life-history stage: Selection on size of hatchling turtles. Ecology 81: 2290-2304.

Jones, T.T., R.D. Reina, C.A. Darveau \& P.L. Lutz. 2007. Ontogeny of energetics in leatherback (Dermochelys coriacea) and olive ridley (Lepidochelys olivacea) sea turtle hatchlings. Comp. Biochem. Phys. A 147: 313-322.

Kawamura, G., T. Naohara, Y. Tanaka, T. Nishi \& K. Anraku. 2009. Near-ultraviolet radiation guides the emerged hatchlings of loggerhead turtles Caretta caretta (Linnaeus) from a nesting beach to the sea at night. Mar. Freshw. Behav. Phy. 42: 19-30.

Koch, A.U., M.L. Guinea \& S.D. Whiting. 2008. Asynchronous emergence of Flatback seaturtles, Natator depressus, from a beach hatchery in Northern Australia. J. Herpetol. 42: 1-8.

Kudo, H., A. Murakami \& S. Watanabe. 2003. Effects of sand hardness and human beach use on emergence success of loggerhead sea turtles on Yakushima Island, Japan. Chelonian Conserv. Bi. 4: 695-696.

Lorne, J.K. \& M. Salmon. 2007. Effects of exposure to artificial lighting on orientation of hatchling sea turtles on the beach and in the ocean. Endangered Species Research 3: 23-30.

Lum, L.L. 2005. Beach. dynamics and nest distribution of the leatherback turtle (Dermochelys coriacea) at Grande Riviere Beach, Trinidad \& Tobago. Rev. Biol. Trop. 53: 239-248.
Maros, A., A. Louveaux, E. Liot, J. Marmet \& M. Girondot. 2005. Identifying characteristics of Scapteriscus spp. (Orthoptera : Gryllotalpidae) apparent predators of marine turtle eggs. Environ. Entomol. 34: 10631070.

Matsuzawa, Y., K. Sato, W. Sakamoto \& K.A. Bjorndal. 2002. Seasonal fluctuations in sand temperature: effects on the incubation period and mortality of loggerhead sea turtle (Caretta caretta) pre-emergent hatchlings in Minabe, Japan. Mar. Biol. 140: 639-646.

Mazaris, A.D., S. Kramer-Schadt, J. Tzanopoulos, K. Johst, G. Matsinos \& J.D. Pantis. 2009. Assessing the relative importance of conservation measures applied on sea turtles: comparison of measures focusing on nesting success and hatching recruitment success. Amphib. Reptil. 30: 221-231.

McGowan, A., A.C. Broderick, J. Deeming, B.J. Godley \& E.G. Hancock. 2001. Dipteran infestation of loggerhead (Caretta caretta) and green (Chelonia mydas) sea turtle nests in northern Cyprus. J. Nat. Hist. 35: 573-581.

Moran, K.L., K.A. Bjorndal \& A.B. Bolten. 1999. Effects of the thermal environment on the temporal pattern of emergence of hatchling loggerhead turtles Caretta caretta. Mar. Ecol. Prog. Ser. 189: 251-261.

Mrosovsky, N. 1968. Nocturnal emergence of hatchling sea turtles: Control by thermal inhibition of activity. Nature 220: 1338-1339.

Mrosovsky, N., S. Kamel, A.F. Rees \& D. Margaritoulis. 2002. Pivotal temperature for loggerhead turtles (Caretta caretta) from Kyparissia Bay, Greece. Can. J. Zool. 80: 2118-2124.

Patino-Martinez, J., A. Marco, L. Quinones \& B. Godley. 2008. Globally significant nesting of the leatherback turtle (Dermochelys coriacea) on the Caribbean coast of Colombia and Panama. Biol. Conserv. 141: 1982-1988

Platt, S.G., T.R. Rainwater, J.B. Thorbjarnarson \& S.T. McMurry. 2008. Reproductive dynamics of a tropical freshwater crocodilian: Morelet's crocodile in northern Belize. Journal of Zoology 275: 177-189.

Quinones, L., J. Patino-Martinez \& A. Marco. 2007. Factores que influyen en la puesta, la incubación y el éxito de eclosión de la tortuga laúd, Dermochelys coriacea, en la Playona, Chocó, Colombia. Rev. Esp. Herp. 21: 5-17.

Reina, R.D., P.A. Mayor, J.R. Spotila, R. Piedra \& F.V. Paladino. 2002. Nesting ecology of the leatherback turtle, Dermochelys coriacea, at Parque Nacional 
Marino Las Baulas, Costa Rica: 1988-1989 to 19992000. Copeia 653-664.

Rimblot, F., J. Fretey, N. Mrosovsky, J. Lescure \& C. Pieau. 1985. Sexual differentiation as a function of the incubation temperature of eggs in the sea-turtle Dermochelys coriacea (Vandelli, 1761). Amphib. Reptil. 6: 83-92.

Spencer, R.J., M.B. Thompson \& P.B. Banks. 2001. Hatch or wait? A dilemma in reptilian incubation. Oikos 93: 401-406.

Spotila, J.R., R.D. Reina, A.C. Steyermark, P.T. Plotkin \& F.V. Paladino. 2000. Pacific leatherback turtles face extinction. Nature 405: 529-530.

Stancyk, S.E. 1995. Non human predators of sea turtles and their control, p . 139-152. In K.A. Bjorndal. Biology and conservation of sea turtles. Smithsonian, Washinton, D. C. EEUU.

Tapilatu, R.F. \& M. Tiwari. 2007. Leatherback turtle, Dermochelys coriacea, hatching success at JamursbaMedi and Wermon beaches in Papua, Indonesia. Chelonian Conserv. Bi. 6: 154-158.
Tucker, J.K., G.L. Paukstis \& F.J. Janzen. 2008. Does predator swamping promote synchronous emergence of turtle hatchlings among nests? Behav. Ecol. 19: 35-40.

Wallace, B.P., P.R. Sotherland, J.R. Spotila, R.D. Reina, B.F. Franks \& F.V. Paladino. 2004. Biotic and abiotic factors affect the nest environment of embryonic Leatherback turtles, Dermochelys coriacea. Physiol. Biochem. Zool. 77: 423-432.

Wallace, B.P., P.R. Sotherland, P.S. Tomillo, S.S. Bouchard, R.D. Reina, J.R. Spotila \& F.V. Paladino. 2006. Egg components, egg size, and hatchling size in Leatherback turtles. Comp. Biochem. Phys. A 145: 524-532.

Whitmore, C.P. \& P.H. Dutton. 1985. Infertility, embryonic mortality and nest-site selection in Leatherback and green sea turtles in Suriname. Biol. Conserv. 34: 251-272.

Witherington, B.E., K.A. Bjorndal \& C.M. McCabe. 1990. Temporal pattern of nocturnal emergence of Loggerhead turtle hatchlings from natural nests. Copeia 1165-1168. 